\section{'Synovial cellular and molecular signatures stratify clinical response to csDMARD therapy and predict radiographic progression in early rheumatoid arthritis patients'}

We read with interest the article by Humby et $a l^{1}$ on the three synovial histological and molecular patterns of rheumatoid arthritis (RA), characterised as predominantly lymphoid, myeloid and fibroid. The body of work emanates from a large and impressive multicentre study with extensive molecular data and showed that in an early, treatment-naïve RA cohort, a fibroid pathological subtype was associated with poorer response to conventional synthetic disease modifying antirheumatic drugs compared with myeloid and lymphoid profiles.

Positive autoantibody status, including disease-specific anticitrullinated peptide antibodies (ACPA), is heavily weighted in the 2010 RA classification criteria. Furthermore, the top 20 genes implicated in ACPA-positive RA, including major histocompatibility complex (MHC) class II associations, PTPN22, CTLA-4 and others, ${ }^{2}$ suggest a sequence of events, whereby non-specific antigen citrullination in peripheral tissues and localised autoimmunity leads to dysregulation in adaptive immunity (played out in the primary and secondary lymphoid organs), systemic autoimmunity and ultimately RA disease manifesting primarily as joint synovitis. In this clinical context, studies evaluating therapies introduced at the earliest stages of disease have been able to demonstrate remarkable efficacy, with some studies over $90 \%$ of cases showing meaningful responses. ${ }^{3}$ If confirmed, the findings of Humby et al could significantly alter the management approach in RA, perhaps suggesting the necessity for synovial biopsy to secure accurate histological and transcriptional characterisation and avoid therapeutic strategies destined to fail, particularly in the fibroblastic RA group. The findings may also suggest the need for novel therapeutic approaches, specifically targeting fibroblasts. The assumption here, however, is that the fibroblastic phenotype represents an RA disease endotype. We would like to postulate that the fibroblastic group may not in all cases represent 'true' RA but rather postinflammation scarring and/ or coexistent osteoarthritis (OA), and we believe this merits evaluation.

Approximately half of the cases in the fibroblastic group were ACPA and rheumatoid factor (RF) negative. This, together with showing the lowest acute phase levels, swollen joint and ultrasound scores (particularly power doppler), and the near complete absence of immune cells, strongly point to a non-RA inflammatory mechanism. The majority of biopsies showing fibroblastic changes came from medium-sized joints (presumably mainly the wrists), with the remainder from small joints. The wrist is a common site of OA, as are the proximal interphalangeal (especially second and third) and metacarpophalangeal joints. The precise age and distribution of joint involvement in the fibroblastic group would thus be enormously instructive to better understand the phenotype. We would be interested to learn if there was clinical evidence of $\mathrm{OA}$ at baseline and/or follow-up in any of these cases. Likewise, was there imaging evidence of OA recorded, such as radiographic changes and/or any suggestion of OA, on ultrasound? With lower swollen joint counts and acute phase reactants, it is perhaps surprising that the two other components of the disease activity score (patient visual analogue score and tender joint count, considered more subjective measures) ${ }^{5}$ appear to be comparable to the lymphomyeloid and diffuse myeloid subtypes. We would be interested to learn of the individual components driving raised disease activity scores in those subjects of fibroblastic phenotype demonstrating the aforementioned low inflammatory clinical profile.

We would acknowledge that half of the fibroblastic group had ACPA or RF-positive status. However, the ACPA titres appeared lower in this group (particularly compared with the lymphomyeloid subset). Given that the mean age of onset of RA is over 50 (reflected in this study), there is the possibility of the coexistence of RA and associated OA; a scenario that is observed in clinical practice and which can be the basis for ongoing diagnostic and treatment dilemmas. Painful OA-related pathology with negative histology for immune cells would certainly offer an explanation for such findings. This important point deserves consideration and could be addressed by procuring MRI scans in the 35 cases of fibroblastic phenotype. Furthermore, we occasionally see RA initially declare itself in osteoarthritic joints. Given that OA is an inflammatory disease and that non-specific inflammation underpins citrullinated neoepitopes, it is plausible that an OA phenotype with its intermittent clinical bouts of swelling could serve as an initiator of autoimmunity and/or RA disease.

To summarise, we would value clarification on the pattern of fibroblastic RA in both the ACPA positive and negative groups. Further information on coexistent or incidental OA would provide significant additional insights and improve our understanding of the proposed fibroblastic phenotype of RA. Maya H Buch $\odot,^{1,2}$ Andrew Melville $\odot,{ }^{1,2}$ Dennis G McGonagle
'Leeds Institute of Rheumatic and Musculoskeletal Medicine, University of Leeds,
Leeds, UK
${ }^{2}$ NIHR Leeds Biomedical Research Centre, Leeds Teaching Hospitals NHS Trust, Leeds,
UK

Correspondence to Professor Maya H Buch, Leeds Institute of Rheumatic and Musculoskeletal Medicine, University of Leeds, Leeds LS2 9JT, UK; m.buch@leeds.ac.uk

Contributors MHB and DGMG: conception of the work, write-up and final approval of the manuscript; AM: write-up and final approval of the manuscript.

Funding The authors have not declared a specific grant for this research from any funding agency in the public, commercial or not-for-profit sectors.

Competing interests None declared.

Patient consent for publication Not required.

Provenance and peer review Not commissioned; internally peer reviewed.

(c) Author(s) (or their employer(s)) 2020. No commercial re-use. See rights and permissions. Published by BMJ.

\section{Check for updates}

To cite Buch MH, Melville A, McGonagle DG. Ann Rheum Dis 2020;79:e140.

Received 12 June 2019

Accepted 17 June 2019

Published Online First 13 July 2019

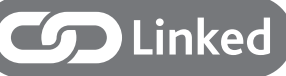

- http://dx.doi.org/10.1136/annrheumdis-2019-215903

Ann Rheum Dis 2020;79:e140. doi:10.1136/annrheumdis-2019-215881

\section{ORCID iDs}

Maya H Buch http://orcid.org/0000-0002-8962-5642

Andrew Melville http://orcid.org/0000-0002-2604-9123 


\section{REFERENCES}

1 Humby F, Lewis M, Ramamoorthi N, et al. Synovial cellular and molecular signatures stratify clinical response to csDMARD therapy and predict radiographic progression in early rheumatoid arthritis patients. Ann Rheum Dis 2019;78:761-72.

2 Yarwood A, Huizinga TWJ, Worthington J. The genetics of rheumatoid arthritis: risk and protection in different stages of the evolution of RA. Rheumatology 2016;55:199-209.
3 Emery P, Hammoudeh M, FitzGerald O, et al. Sustained remission with etanercept tapering in early rheumatoid arthritis. N Engl J Med 2014;371:1781-92.

4 Emery P, Kvien TK, Combe B, et al. Combination etanercept and methotrexate provides better disease control in very early ( $<=4$ months) versus early rheumatoid arthritis ( $>4$ months and $<2$ years): post hoc analyses from the COMET study. Ann Rheum Dis 2012;71:989-92.

5 Hensor EMA, McKeigue P, Ling SF, et al. Validity of a two-component imagingderived disease activity score for improved assessment of synovitis in early rheumatoid arthritis. Rheumatology 2019;49:pii: kez049. 